\title{
Coping with Complexity When Predicting Surface Roughness in Milling Processes: Hybrid Incremental Model with Optimal Parametrization
}

\author{
Gerardo Beruvides, ${ }^{1}$ Fernando Castaño, ${ }^{1}$ Rodolfo E. Haber, ${ }^{1}$ \\ Ramón Quiza, ${ }^{2}$ and Alberto Villalonga ${ }^{2}$ \\ ${ }^{1}$ Centre for Automation and Robotics, UPM-CSIC, Arganda del Rey, Spain \\ ${ }^{2}$ Research Group on Advanced and Sustainable Manufacturing, UM, Matanzas, Cuba \\ Correspondence should be addressed to Rodolfo E. Haber; rodolfo.haber@car.upm-csic.es
}

Received 26 September 2017; Accepted 27 November 2017; Published 17 December 2017

Academic Editor: Rosario Domingo

Copyright (C) 2017 Gerardo Beruvides et al. This is an open access article distributed under the Creative Commons Attribution License, which permits unrestricted use, distribution, and reproduction in any medium, provided the original work is properly cited.

\begin{abstract}
The complexity of machining processes relies on the inherent physical mechanisms governing these processes including nonlinear, emergent, and time-variant behavior. The measurement of surface roughness is a critical step done offline by expensive quality control procedures. The surface roughness prediction using an online efficient computational method is a difficult task due to the complexity of machining processes. The paradigm of hybrid incremental modeling makes it possible to address the complexity and nonlinear behavior of machining processes. Parametrization of models is, however, one bottleneck for full deployment of solutions, and the optimal setting of model parameters becomes an essential task. This paper presents a method based on simulated annealing for optimal parameters tuning of the hybrid incremental model. The hybrid incremental modeling plus simulated annealing is applied for predicting the surface roughness in milling processes. Two comparative studies to assess the accuracy and overall quality of the proposed strategy are carried out. The first comparative demonstrates that the proposed strategy is more accurate than theoretical, energy-based, and Taguchi models for predicting surface roughness. The second study also corroborates that hybrid incremental model plus simulated annealing is better than a Bayesian network and a multilayer perceptron for correctly predicting the surface roughness.
\end{abstract}

\section{Introduction}

Despite the progress in science and technology, manufacturing processes are still considered complex systems consisting of several interacting subsystems with noisy, timevariant, and nonlinear behavior $[1,2]$. New models are then required to better deal with manufacturing companies' threats innovating in whole value chain [3]. Machining processes, and specifically milling processes, are key elements of manufacturing value chain [4]. The roots of the machining complexity rely on the inherent physical mechanisms governing the process itself. Different modeling techniques have been explored to describe and understand the geometric and physical characteristics of the cutting process $[5,6]$.

Quality monitoring is still the cornerstone and the bottleneck of many industrial processes [7]. For some manufacturing companies centered on aeronautical and automotive sectors, parts quality should fulfill strict requirements for dimensional $[8,9]$ and surface quality [10]. Inappropriate surface quality leads to removing defective parts from the production line and remanufacturing the component [11]. It implies more energy consumption of machine tools and more scraps resulting from the machining processes. In-process solution for predicting quality variables is in the agenda of many companies, and many solutions are being explored to decrease the environmental impact of machining processes due to poor surface quality of parts [12].

Surface roughness prediction studies in end milling operations are usually based on three main parameters composed of cutting speed, feed rate, and depth of cut [13]. Surface roughness and specific cutting energy consumption in slot 
milling are very related [14]. Recent studies demonstrate that net cutting specific energy is an effective process signature for surface integrity [15].

The review of traditional and emergent modeling strategies for manufacturing processes is out the scope of this paper. Nevertheless, it is important to remark that artificial neural networks (ANN) and fuzzy systems represent the most widely applied artificial intelligence techniques for modeling, control, and supervision [16, 17]. A thorough revision of computational intelligence techniques is out of the scope of this paper. The potential of artificial neural networks is often combined with the ability of fuzzy systems to represent human thought and robustness in the presence of noise and process uncertainty [18]. Fuzzy systems can also be combined with classical modeling techniques in order to better capture the most important characteristics of processes [19].

On the other hand, among model-free methods reported in the literature, the incremental models are proposed in [20]. Incrementally, principle can be fully exploited starting from a simple and generic form of the model. Further refining procedures can be iteratively applied using some more localized techniques to represent specific regions of the input space. This work is focused on that principle for developing a hybrid incremental model inspired in [21] and then presents a case study for surface roughness prediction in milling processes. Surface roughness is influenced by tool wear, chattering, inappropriate cutting conditions, and so forth, and therefore it is mainly used to monitor the quality of the manufactured components [22].

In this work, a regression technique is applied for fitting the global characteristics whereas the fuzzy $k$-nearestneighbors algorithm [23] is selected for the subsequent refinement of the global model. The main advantages of fuzzy $k$-nearest-neighbors algorithm have been already demonstrated in various studies [24, 25]. Noticeably, none of previous works has evaluated the capability of this instancebased learning method in surface roughness prediction.

However, setting parameters of the hybrid incremental model (HIM) is not an easy task because it strongly depends on the case study. Optimal tuning of parameters requires one strategy for optimization among all gradient-free techniques [26]. Simulated annealing (SA) is a probabilistic hill-climbing technique that emulates the physical annealing and cooling process of metals, based on a simple criterion that searches the problem space by piecewise perturbations of the estimates of the parameters that are being optimized [27]. SA has been used widely as optimization technique in many fields from optimal task allocation in manufacturing systems to vehicle routing systems [28].

From the best of authors' knowledge, the main contributions of this work are twofold. Firstly, a model-based procedure is implemented for predicting surface roughness based on a hybrid incremental modeling strategy. Secondly, a wellknown optimization method with proven convergence properties is applied to achieve the optimal parametrization for the HIM method. The performance of the proposed method is assessed by means of two comparative studies. Firstly, the prediction capability is analyzed with regard to a theoretical, energy-based, and Taguchi models. Secondly, four errorbased criteria are applied to perform the comparison with a Bayesian network and a multilayer perceptron for predicting surface roughness. The suitability of the proposed approach for minimizing the number of parts to be remanufactured due to poor surface roughness is then demonstrated. The rest of the paper is organized as follows. Section 2 presents a description of hybrid incremental modeling. The experimental platform and the comparative study are presented in Section 3. Conclusions are shown in Section 4.

\section{Hybrid Incremental Modeling Based on the Optimal Setting}

The behavior of a locally nonlinear system [21] can be captured by hybrid incremental modeling where a global model represents the general behavior of the system and the local model shows the local behavior. Black box approach is an appropriate strategy when no prior knowledge of the system is available. For the sake of simplicity and clarity, a linear regression is selected for representing the global behavior of the system. Therefore, least squares are chosen to fit a polynomial of degree $m$ with the following output:

$$
\widehat{y}_{B}\left(x_{i}\right)=f_{B}\left(x_{i}, g\left(x_{i}\right)\right),
$$

where $x_{i}$ is the $i$ th input data and $g\left(x_{i}\right)$ is its output value.

Therefore, the global model consists in the computed parameters of the fitting function (i.e., the $m$-degree polynomial).

The development of the local model requires a simple and easily interpretable technique from the viewpoint of industrial informatics. Data normalization [29] is carried out because different variable ranges affect negatively the performance of the algorithms. On the other hand, fuzzy $k$-Nearest Neighbors (F-kNN) approach has the required characteristics justifying its selection [30]. The similarity between $q$ and the points of $N$ is given by

$$
\begin{aligned}
& S\left(n_{i}, q\right) \\
& = \begin{cases}1, & \text { if }\left\|n_{i}-q\right\|=0, \\
{\left[\left\|n_{i}-q\right\|^{2 /(p-1)} \cdot \sum_{j=1}^{k}\left(\frac{1}{\left\|n_{j}-q\right\|}\right)^{2 /(p-1)}\right]^{-1}} & \text { if }\left\|n_{i}-q\right\| \neq 0,\end{cases}
\end{aligned}
$$

where $n_{i}$ is the $i$ th neighbor of the query point $q$ and $p$ is the fuzzy strength parameter.

The target value of query point $q$ is now calculated as the mean of the target values of the points of the set $N$, weighted by the similarity $S$ :

$$
\widehat{g}(q)=\sum_{i=0}^{k} S\left(n_{i}, q\right) \cdot g\left(n_{i}\right) .
$$

The incremental model integrates the global and local models described above. Thus, let $\widehat{y}_{B}(x)$ be the function that is the output of the basic model. Then the prediction error of the basic model is

$$
\varepsilon(x)=g(x)-\widehat{y}_{B}(x) .
$$


It is important to remark that F-kNN only use errors resulting from the global modeling strategy. Therefore, the refinement of the global model output in regions with localized nonlinear behavior is done by F-kNN.

The incremental model evaluates a sample of data input $q$, by adding to the output of the basic model the compensation term calculated by the local model according to (4):

$$
\hat{y}(q)=\hat{y}_{B}(q)+\hat{g}(q) .
$$

The tuning parameters of hybrid incremental model, namely, degree of the polynomial $(m)$, the neighborhood size $(k)$, and the fuzzy strength $(p)$, play an important role in setting the predictive model. These parameters influence the model's performance, and they are required to be properly computed to produce the best output estimation using new input data.

Simulated annealing (SA) is selected among the arsenal of gradient-free techniques to search for an optimal set of model parameters. The main rationale for its selection relies on it easy-to-implement probabilistic algorithm able to yield very good solutions for a wide variety of problems. A thorough study on the fast convergence and relatively low complexity of the algorithm is out of the scope of this paper but it has been recently reported [31]. The SA algorithm starts with initial modeling parameters $K_{\mathrm{HIM}}=\left[m_{0}, k_{0}, p_{0}\right]$ and evaluates the MAPE performance index.

$$
\begin{aligned}
\text { MAPE } & =\frac{1}{N} \sum_{i}^{N}\left|\frac{y_{i}-\hat{y}_{i}}{y_{i}}\right| \cdot 100, \\
K_{\mathrm{HIM}} & =[m, k, p]_{\mathrm{opt}}=\arg \min (\mathrm{MAPE}) .
\end{aligned}
$$

The current modeling parameters $K_{\mathrm{HIM}}$ are perturbed to generate another $\mathrm{K}_{\mathrm{HIM}(\mathrm{NEW})}$ and the mean absolute percentage error (MAPE) performance index is evaluated again. The Metropolis algorithm is the basement for the acceptance and rejection criterion. SA simulates the annealing process as it searches for a solution. A random perturbation is generated on the design variables $m, k, p$ which generates a change in the objective function (i.e., MAPE performance index). These perturbations depend on a temperature index, $T$, and rate at which it is lowered $(\alpha=[0.5,0.99])$. More details on this temperature control parameter for simulated annealing are presented in [32].

Figure 1 represents the temperature index decreasing per iteration of the algorithm. Each set of model parameters $K_{\mathrm{HIM}}$ obtained by this method is substituted into the hybrid incremental model (global plus local models) and the performance of the resulting solution is assessed by means of whole simulation of the system. Further details can be found in [33].

Finally, the MAPE performance index is evaluated by comparing the simulated responses with the desired responses and calculating the MAPE performance index. If the performance index is lower than the previous best performance index, then the new parameters replace the previous parameters. Otherwise, the new model parameters are not immediately discarded.

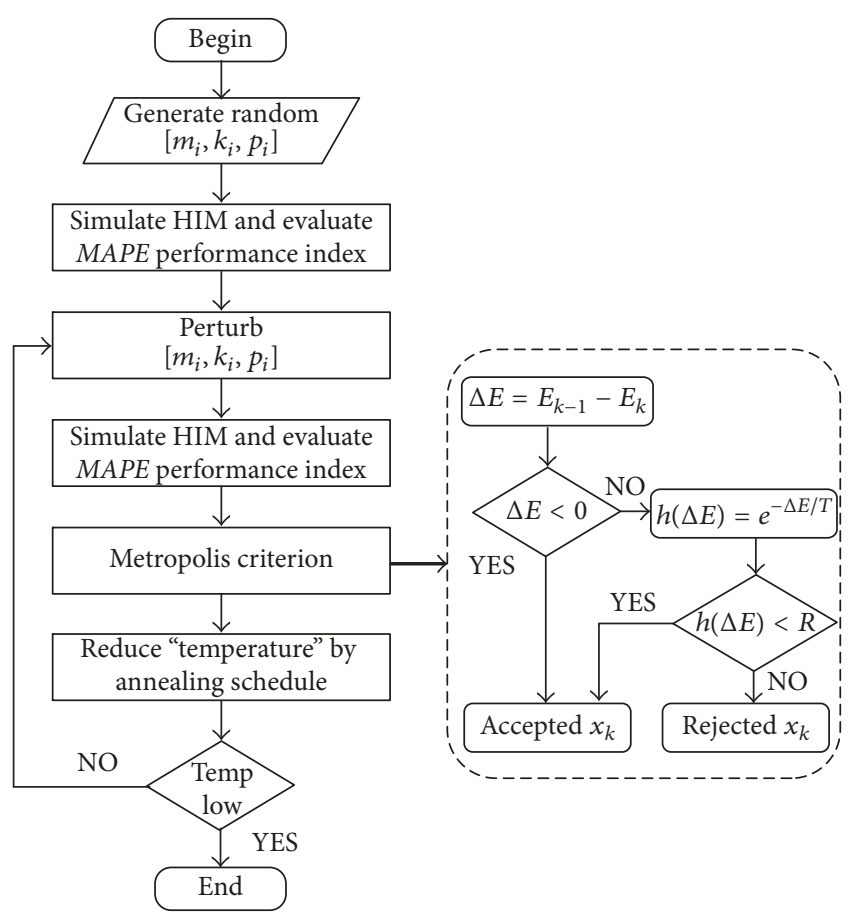

FIGURE 1: Algorithm for optimal tuning of hybrid modeling parameters based on simulated annealing.

The probability, $P$, of the new parameters' cost $\left(\mathrm{MAPE}_{\mathrm{NEW}}\right)$ relative to the previous best cost $\left(\mathrm{MAPE}_{\mathrm{PREV}}\right)$ is calculated using Boltzmann's equation:

$$
P=e^{\left(\mathrm{MAPE}_{\mathrm{PREV}}-\mathrm{MAPE}_{\mathrm{NEW}}\right) / T} .
$$

The temperature index is then reduced by the annealing schedule using a reduction constant. The whole process is repeated until either the MAPE performance index has reached an acceptable minimum level or the temperature value has become too small to perturb the parameters. The main goal is to derive optimal modeling parameters that yield a fast and accurate model through the minimization of the MAPE performance index. Figure 2 shows the training procedure of the model using the target data. Figure 3 depicts how the resulting model estimates or predicts the corresponding output using the input data.

\section{Experimental Study}

Kondia HS1000 machining center equipped with a Siemens $840 \mathrm{D}$ open-architecture $\mathrm{CNC}$ was used in experiments (see Figure 4). In all the cases, $170 \times 100 \times 25$ aluminum AL7075T6 (UNS A97075) workpieces were used with hardness ranging from 65 to 152 Brinell. This material is commonly used in automotive and aeronautical applications.

Figure 5 illustrates the two geometry forms: (b) pockets and (d) island selected for experiments. For a better understanding, a constant spiral strategy was selected for the pocket elaboration and a Morph spiral strategy for the island form. Figure 5 also shows the simulation and cutting trajectories for the pocket (a) and the island (c), as well as, 


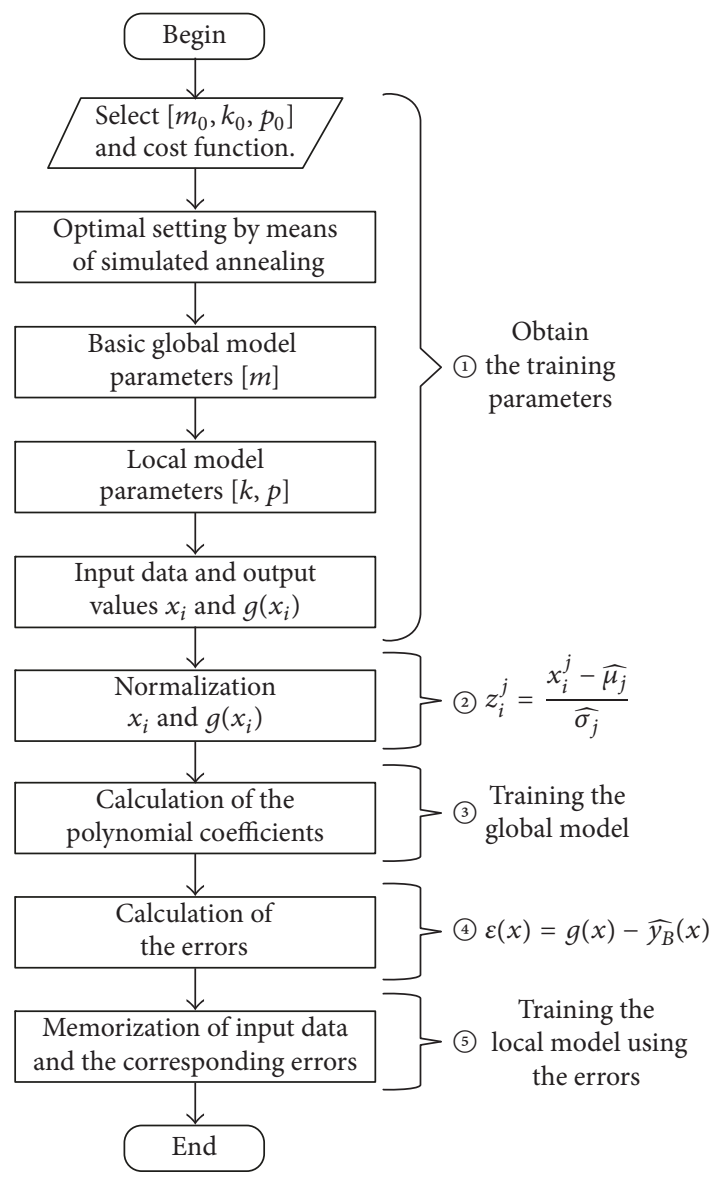

FIGURE 2: Diagram of the procedure for training hybrid incremental model.

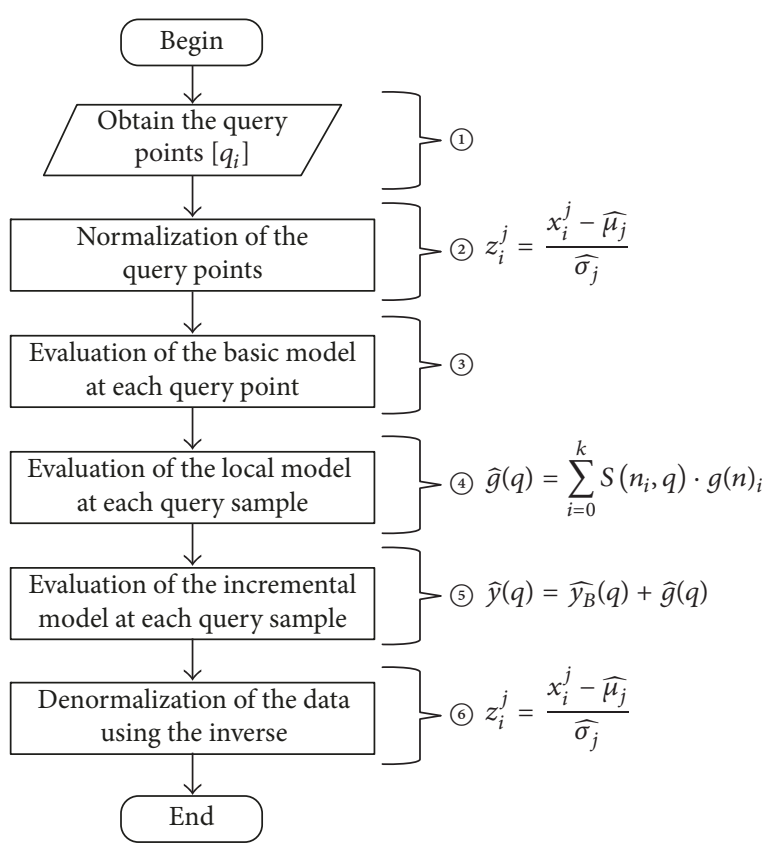

Figure 3: Diagram for executing the hybrid incremental model.

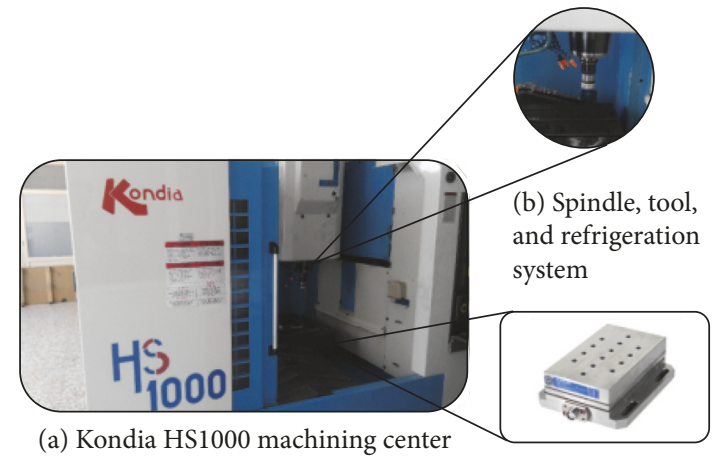

(c) Kistler 9257B dynamometer

FIGURE 4: Kondia HS1000 machining center and sensoring equipment.

the final workpieces (b) pocket \& (d) island after the milling process. In all the cases, the strategies, tool compensations, cutting regimens, and machining time were designed and simulated in Computer-Aided Design and Computer-Aided Manufacturing (CAD/CAM) software and, finally, exported to the CNC machine center.

Table 1 shows the six variables selected in the experimental setup. On one hand, feed rate $\left(f_{z}\right)$, tool diameter $(D)$, radial depth of cut $(a e)$, and spindle rotation speed $(\mathrm{rpm})$ were chosen for their influence on productivity, having direct relationship with key cutting variables such as cutting speed, cutting feed rate, and material removal rate, among others. On the other hand, the geometry curvature (Geom) and material hardness $(H B)$ are much related to appropriate tool selection and the corresponding cutting strategies. For this reason, these six parameters were selected as key process indicators to model the milling process. Furthermore, the machining time for the different geometry is included in Table 1 .

The most used index to characterize the surface roughness is the roughness average, $R a$, that represents the arithmetic mean of the absolute ordinate values $G(w)$ within a sampling length $(L)$ as follows:

$$
R a=\frac{1}{L} \int_{0}^{L}|G(w)| d w
$$

One interesting indicator related to surface roughness is the specific cutting energy consumption (SCEC) introduced by Liu et al. [14]. SCEC is analytically calculated based on the cutting parameters and tool-workpiece couple. SCEC is defined as the cutting energy consumed at the tool tip to remove $1 \mathrm{~mm}^{3}$ of material. Following the definition, the equation for a slotting milling process using a flat-end mill can be estimated as follows:

$$
\begin{aligned}
& \text { SCEC }=\frac{\bar{P}_{n}}{\operatorname{MRR}} \\
& =\sum_{1}^{N_{f}} \int_{0}^{2 \pi} \int_{z_{i 1}}^{z_{i 2}}\left(\frac{n \cdot K t e}{2000 \cdot f \cdot a_{p} \cdot \cos i_{0}}+\frac{K t c}{2000 \cdot N_{f} \cdot a_{p}}\right) d z d \psi,
\end{aligned}
$$




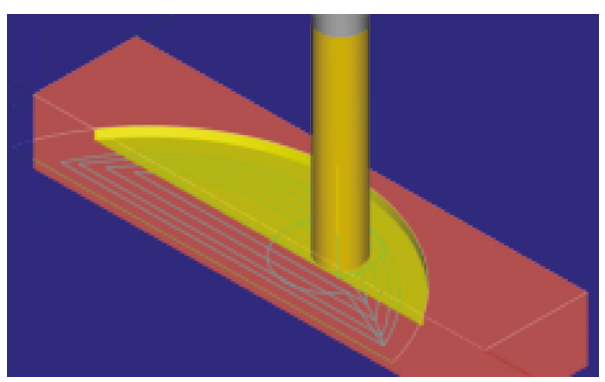

(a) Pocket simulation and trajectory

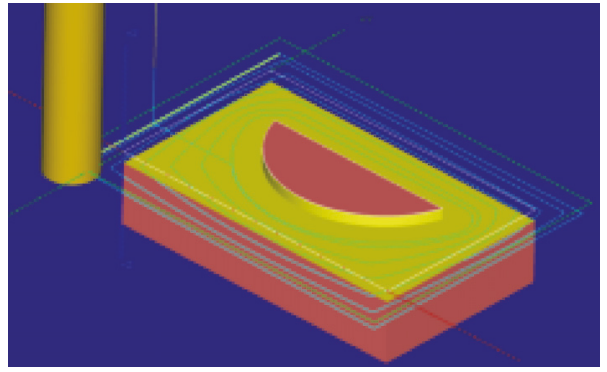

(c) Island simulation and trajectory

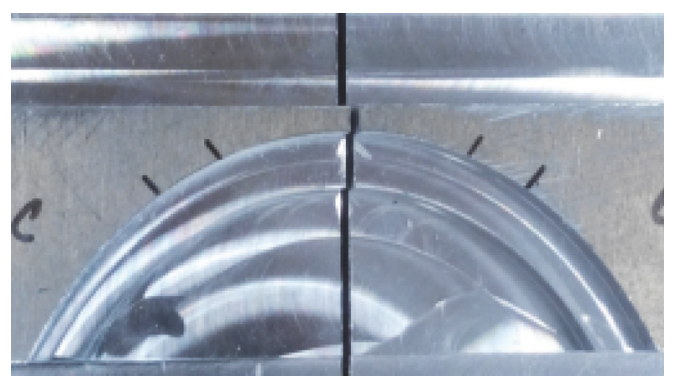

(b) Pocket

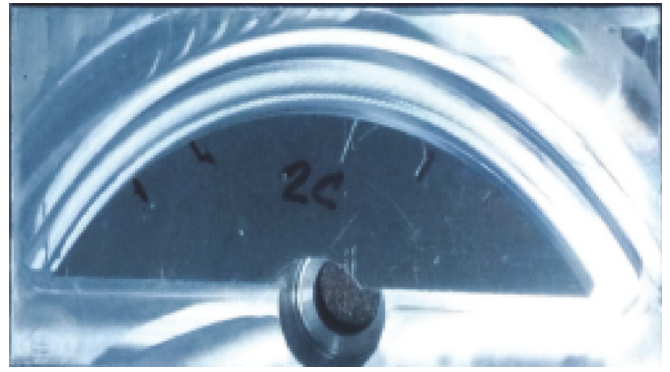

(d) Island

FIGURE 5: Different geometry forms used during the experimentation.

TABLE 1: Factors and factor levels used in the DoE in experiment.

\begin{tabular}{|c|c|c|c|c|c|c|}
\hline $\begin{array}{l}D \\
(\mathrm{~mm})\end{array}$ & $\begin{array}{c}f z \\
\text { (mm/tooth) }\end{array}$ & $\begin{array}{c}a e \\
(\mathrm{~mm})\end{array}$ & $\begin{array}{c}H B \\
\text { (Brinell) } \\
\end{array}$ & $\begin{array}{l}\text { Geom } \\
(\mathrm{mm})\end{array}$ & $\begin{array}{c}\mathrm{rpm} \\
\text { (rev/min) }\end{array}$ & $\begin{array}{c}\text { Machining time } \\
\text { (s) }\end{array}$ \\
\hline 8 & 0.075 & 3 & 94 & 0 & 22500 & 1038 (island); 1212 (pocket) \\
\hline \multirow{2}{*}{10} & 0.050 & 2 & 92 & +0.021 & \multirow{2}{*}{18000} & 1150 (island); 479 (pocket) \\
\hline & 0.100 & 4 & 145 & -0.021 & & 658 (island); 476 (pocket) \\
\hline \multirow{3}{*}{12} & 0.025 & 1 & 67 & +0.042 & \multirow{3}{*}{18000} & 1754 (island); 734 (pocket) \\
\hline & 0.075 & 3 & 94 & 0 & & 675 (island); 253 (pocket) \\
\hline & 0.130 & 5 & 152 & -0.042 & & 445 (island); 152 (pocket) \\
\hline \multirow{2}{*}{16} & 0.050 & 2 & 92 & +0.021 & \multirow{2}{*}{18000} & 685 (island); 258 (pocket) \\
\hline & 0.100 & 4 & 145 & -0.021 & & 666 (island); 141 (pocket) \\
\hline 20 & 0.075 & 3 & 94 & 0 & 15000 & 507 (island); 127 (pocket) \\
\hline
\end{tabular}

where $\bar{P}_{n}$ is the average rotation power; Kte and Ktc are the tangential specific cutting and edge force coefficients; $i_{0}$ is the helix angle; $N_{f}$ is the number of flutes of the flat-end mill; $z_{i 1}$ and $z_{i 2}$ are the lowest and highest position of the infinitesimal cutting edges engaged in cutting in axial direction on the $i$ th flute, respectively.

In order to match the model output with the actual industrial requirements and standards, roughness labels were also allocated according to the average value of surface roughness $(\mu \mathrm{m})$ defined in ISO:1302 (2002) [34]. Table 2 shows variables and the respective assigned intervals for surface roughness from mirror to smooth.

Additionally, the following cutting parameters were also considered as inputs: spindle speed $(\mathrm{rpm})$, feed rate $\left(f_{Z}\right)$, and the resulting forces applied to all directions of cutting plane $\left(F_{x y}\right)$. The cutting forces were measured using a Kistler 9257B dynamometer. The surface roughness $R a$ was measured with
TABLE 2: Variables and intervals assigned.

\begin{tabular}{lcc}
\hline State & $\begin{array}{c}F_{x y} \\
(\mathrm{~N})\end{array}$ & $\begin{array}{c}R a \\
(\mu \mathrm{m})\end{array}$ \\
\hline 1 & $(22,100)$ & Mabel (lower, upper) \\
2 & $(101,208)$ & Mirror $(0.10,0.25)$ \\
3 & $(209,324)$ & Polished $(0.25,0.35)$ \\
4 & $(324,488)$ & Ground $(0.35,0.75)$ \\
\hline
\end{tabular}

the Carl Zeiss Surfcom 130 stylus profilometer. Overall, 992 training samples were used in order to build the model, whereas 72958 test samples were used for the validation study. The average value of surface roughness, $R a$, was chosen as the output variable of the model and it was the only variable offline measured. 

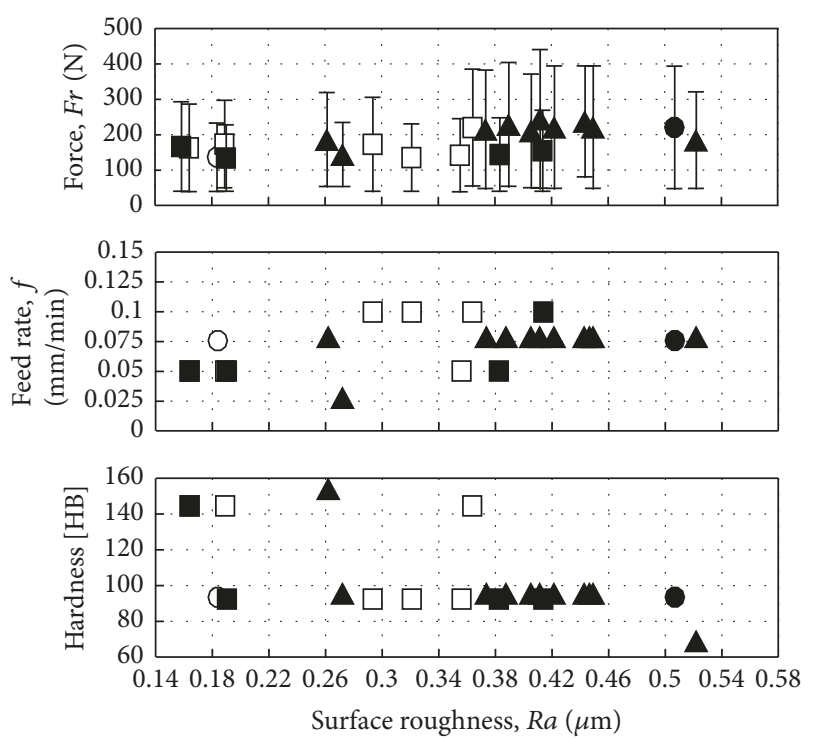

$$
\begin{aligned}
& \text { - } D=8 \quad \square D=16 \\
& \text { 口 } D=10 \quad O D=20 \\
& \text { A } D=12
\end{aligned}
$$

FIGURE 6: Relationship between the plane force, feed rate, and hardness with surface roughness for different diameters.

Figure 6 represents the influence of resultant force, feed rate, and hardness on surface roughness for each tool diameter considered in the study. According to the depicted results, the richness of the training data guarantees that the four roughness levels are well taken into account to address the modeling task.

\section{Experimental Results}

4.1. Optimal Model Configuration. The hybrid incremental model plus simulated annealing procedure (HIM + SA) is implemented as follows. The SA algorithm parameters are reduction constant $\alpha=0.99$ and initial temperature $T_{i}=5000$. Additionally, the number of proposed random perturbations at each temperature is 100 and the number of accepted solutions to proposed random perturbations is 30 . The exploration space is $m \in[1,10], k \in[1,10], p \in[1,3]$ where $m, k \in \mathbb{Z}$ and $p \in \mathbb{R}$.

In order to achieve an unbiased comparison for execution time of the algorithms, all algorithms run with a personal computer having Intel Core i5-3317U CPU $1.70 \mathrm{GHz} 4 \mathrm{~GB}$ RAM. One hundred executions are run. The optimization process lasts $14 \mathrm{CPU}$ seconds. The optimal parameters $(m, k, p)=(2,2,1.92)$ were obtained on the basis of the procedure described in Section 2 by simulated annealing optimization procedure (see Figure 3).

This means a second-order polynomial (linear), two neighbors for the neighborhood size, and a value of 1.92 for the fuzzy strength parameter. The global model is obtained after the training. The errors (residuals) obtained in the training phase are recorded and then they are used during the evaluation by means of (4). The output of the model is

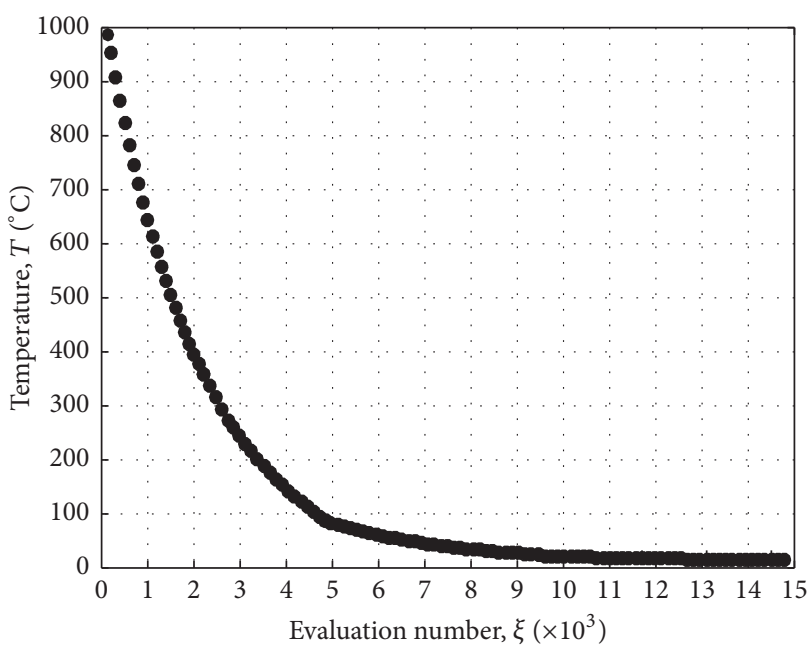

FIgURE 7: Behavior of the temperature in relation to iterations.

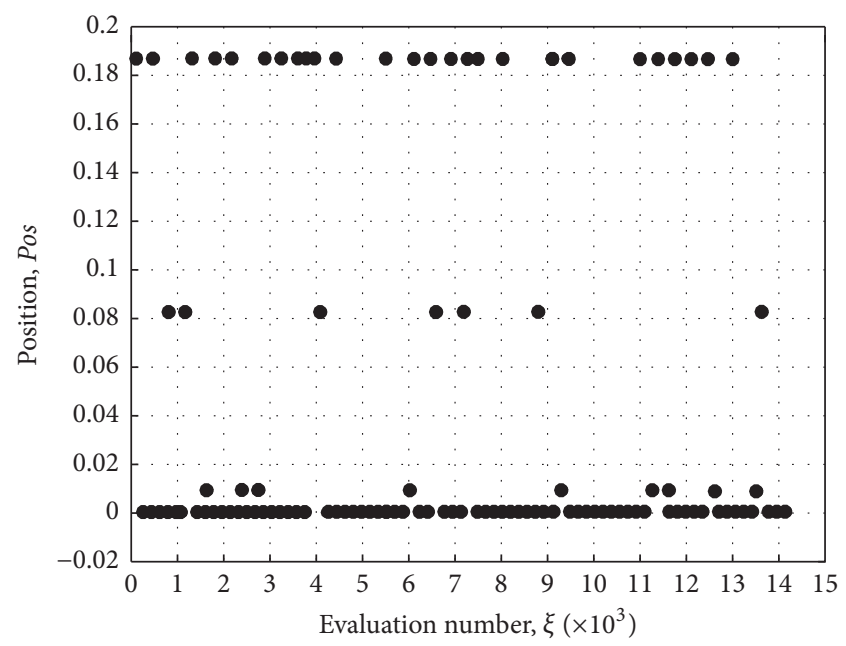

FIGURE 8: Behavior of the cluster center position with regard to iterations.

obtained by applying (5). The minimum of MAPE performance index is $0.88 \%$. The behavior of temperature $(T)$ with regard to the evaluation number $(\xi)$ to obtain the optimal solution is shown in Figure 7. It is clearly shown how after 10,000 evaluations the algorithm converges to a stable region.

Another interesting indicator is the behavior of cluster center position (Pos) with regard to the evaluation number shown in Figure 8. There are two main regions, one close to 0 value and the other close to 0.19 . Furthermore, in some iterations, the cluster center is located at 0.08 or near to 0 .

4.2. Discussion on Surface Roughness Prediction Problems. A comparison with three prediction models reported in the literature is performed to assess the accuracy of the proposed model. According to Wang and Chang [35], the theoretical 
$R a$ estimation for a slotted surface using a flat-end mill can be calculated as

$$
R a^{\kappa}=\frac{f}{4 \cdot \cot \kappa},
$$

where $\kappa$ is the concavity angle ( $k=2.5$ by measurement).

The second model was extracted from the empirical representation surface roughness depending on specific cutting energy consumption (SCEC) index proposed by Liu et al. [14], represented by

$$
R a^{\mathrm{SCEC}}=C_{1} \cdot e^{\mathrm{C}_{2} \cdot \mathrm{SCEC}},
$$

where $C_{1}$ and $C_{2}$ are empirical coefficients estimated in function of $a p$.

Finally, Taguchi methodology is a widely used approach to optimize the parameters for obtaining the minimum surface roughness, and the following representation is also taken from Liu et al. [14]:

$$
R a=10^{-\eta^{*} / 20},
$$

where $\eta^{*}$ is the predicted $S / N$ ratio for the predicted cutting condition.

For the sake of coherence with the above-mentioned works, the performance index or figure of merit used to carry out a first comparison is the prediction relative error. Table 3 shows the prediction relative errors using models (see (10)-(12)) and HIM + SA.

This first comparative study aims at assessing the accuracy of the four models considered in the comparison. The column labelled $R a$ represents the nominal values measured in workpieces and the subscripts ( $\kappa$, SCEC, $\eta$, HIM) represent the predicted values using the models (see (10)-(12)). The theoretical model, the SCEC model, and the Taguchi model are evaluated using the cutting conditions already reported in $[14,35]$ with $r p m \in[1000,3000](\mathrm{rpm}), a e \in[1,3](\mathrm{mm})$, $f \in[50,100](\mathrm{mm} / \mathrm{min})$. In the work herein reported the same material (AL-7075) and the same tool (a 2-flute solid carbide flat-end mill with a diameter of $10 \mathrm{~mm}$ ) are also used in the experiments. However, the depth of cut, the cutting speed, and the feed rate in our experiments (see Table 1) are higher than the cutting conditions reported in [14, 35]. In order to assess the precision of the four models in their respective cutting conditions, the predicted and measured values of surface roughness are compared. HIM + SA yields the best fitting between the predicted and measured values of surface roughness with best prediction errors.

Figure 9 depicts the graphical representation of measured versus predicted $\mathrm{Ra}$ for all models considered in this comparison. HIM + SA shows the best fit in all the cases, although it is important to highlight that SCEC model also shows very good behavior. From these results we can conclude that a clear representation of the milling process can be obtained with the proposed method, being a powerful tool to predict in-process surface roughness based on the cutting parameters.

In the literature, there are plenty of artificial intelligence techniques applied to surface roughness detection and prediction. For the sake of clarity, two modeling strategies based

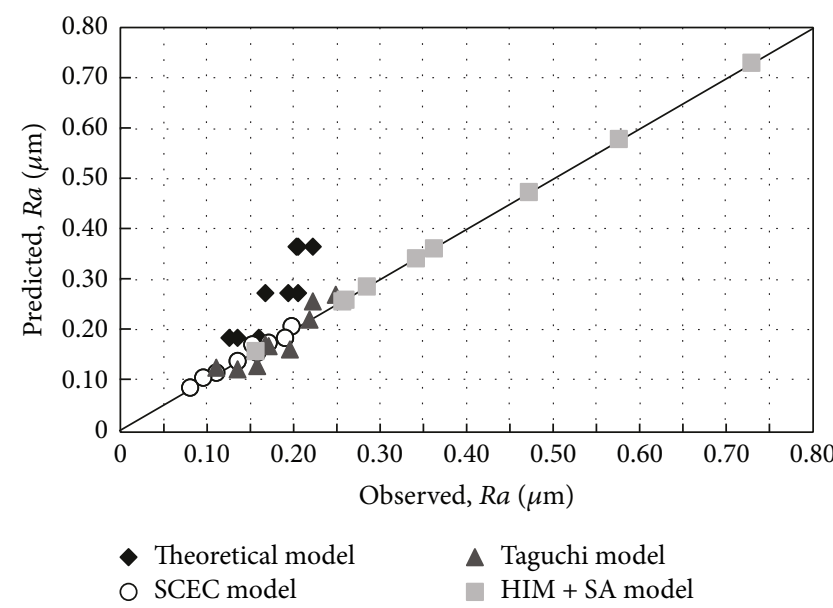

FIGURE 9: Comparison between observed versus predicted for the studied models.

on a Bayesian network (BN) and a multilayer perceptron (MLP) are also compared with the HIM + SA. In order to carry out a thorough evaluation of the model, four errorbased performance indices and two classification criteria are considered in this study. Therefore, in the second comparative study, a total of six performance indices were applied to assess the actual behavior on the basis of experimental run as follows: number of parts correctly manufactured (NPM), the number of parts to be remanufactured (NPR), the mean absolute error (MAE), the root mean squared error (RMSE), the relative absolute error (RAE), and the root relative squared error (RRSE).

Table 4 shows the main characteristics of the three approaches considered in the second comparative study. Multilayer perceptron (MLP) is one of the most widely applied neural networks at industrial level. However, the main drawback is related to the setting and tuning of network parameters such as number of hidden layers, number of nodes in the hidden layer(s), and form of activation functions. On the contrary, BNs have an easy and fast construction procedure without tuning parameters. The rationale for the good interpretability of BNs is indeed the reasoning based on real-world models.

However, memory requirements might limit the transfers of knowledge and real-time applications. Further details on designing issues are reported in [36]. HIM + SA has some interesting features such as simple structure and easy training with few tuning parameters enabled by an optimal setting procedure (simulated annealing). $\mathrm{HIM}+\mathrm{SA}$ also outperforms BN and MLP in terms of computing time. BN requires $0.08 \mathrm{CPU}$ seconds, MLP requires $12.69 \mathrm{CPU}$ seconds and $\mathrm{HIM}+\mathrm{SA}$ requires $0.01 \mathrm{CPU}$ seconds.

Table 5 shows the results of the second comparative study. The application of the BN and ANN yielded RAE of $10.41 \%$ and $13.05 \%$, respectively. On the contrary, HIM + SA achieved an excellent accuracy, for instance, $2.68 \%$ in RAE, almost five times less error than the BN. The excellent behavior is also endorsed with the best number of parts correctly manufactured of $98.88 \%$. Overall, HIM + SA outperforms 


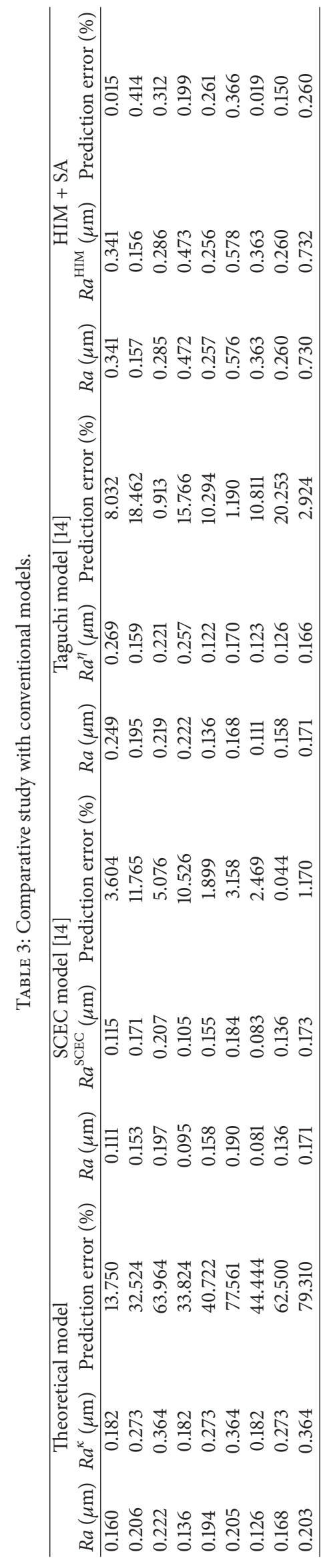


TABLE 4: Main parameters of each modeling strategy considered in this study.

\begin{tabular}{lccc}
\hline Algorithms & BN & ANN & HIM + SA \\
\hline Model & $\begin{array}{c}\text { Seven inputs } \\
(F r, f z, D, a e, H B, G e o m, r p m) \\
\text { Output: } R a\end{array}$ & $\begin{array}{c}\text { Seven inputs: }(F r, f z, D, a e, H B, \\
\text { Geom, } r p m) \\
\text { Output: } R a\end{array}$ & $\begin{array}{c}\text { Seven inputs } \\
(F r, f z, D, a e, H B, G e o m, r p m) \\
\text { Output: } R a\end{array}$ \\
\hline Clustering & No (TAN) & No (BP) & Fuzzy $k$-NN \\
Structure & Three layers (4-11-4 neurons) & Tree-like structure & $\begin{array}{c}\text { Second-order polynomial, two } \\
\text { neighbors, and 1.92 for fuzzy strength } \\
\text { parameter }\end{array}$ \\
\hline Inference & Global & Causal and abductive & Global and local \\
\hline Main parameters to adjust & No tuning parameters & Many (MLP) & m, $k, p$, optimal setting by SA \\
\hline Training algorithms & No (estimation of conditional & BP & LSE + IBL \\
\hline Interpretability & Yes & No & Partially
\end{tabular}

BN: Bayesian network; ANN: artificial neural network; HIM + SA: hybrid incremental model with simulated annealing; TAN: tree augmented Naive-Bayes; BP: backpropagation; MLP: multilayer perceptron; LSE: least square error; IBL: instance based learning.

TABLE 5: Comparative study of the three models (ANN, BN, HIM + SA).

\begin{tabular}{lccc}
\hline Performance index/approach & BN & ANN & HIM + SA \\
\hline NPM (\%) & 96.35 & 94.84 & 98.88 \\
NPR (\%) & 3.65 & 5.16 & 1.12 \\
MAE & 0.03 & 0.04 & 0.0032 \\
RMSE & 3.65 & 5.16 & 0.0227 \\
RAE (\%) & 10.41 & 13.05 & 2.68 \\
RRSE (\%) & 32.66 & 33.70 & 4.77 \\
\hline
\end{tabular}

significantly BN and ANN with regard to all figures of merits considered in this second study; specifically the number of parts to be remanufactured is the least value of all.

\section{Conclusions}

The development of adequate modeling strategies to deal with complexity of machining processes is a must. The prediction of surface quality is not straightforward. The need of decreasing parts to be remanufactured due to poor surface quality is motivated by the direct impact on economy and environment. This paper presents a hybrid incremental modeling strategy with an optimal setting procedure for quality detection in milling process. The procedure for designing and implementing hybrid incremental models with optimal parameters is simple and computationally efficient. The study demonstrates how surface roughness is predicted with a basic parameters configuration (second-order polynomial, two neighbors, and the fuzzy strength parameter near to two) using a simulated annealing optimization method.

Two comparative studies with traditional techniques (i.e., theoretical model, energy model, and Taguchi-based model) and artificial intelligence-based techniques (i.e., a multilayer perceptron and a Bayesian network) for predicting surface roughness have demonstrated that the proposed strategy outperforms significantly the others techniques and models considered in this study. The number of parts to be remanufactured using hybrid incremental model with optimal parametrization is less than applying either, a multilayer perceptron and a Bayesian network (e.g., about one percentage). The hybrid incremental model yields also better error-based performance indices for predicting the surface roughness than above-mentioned Bayesian and neural network models.

Hybrid incremental modeling plus simulated annealing for optimal parametrization can be extended to a wide range of manufacturing processes for estimating part quality. Further studies will be conducted to relate model outputs to other variables such as current and power consumption.

\section{Conflicts of Interest}

The authors declare that there are no conflicts of interest regarding the publication of this paper.

\section{Acknowledgments}

The work reported in this paper is partially supported by the European Project "IoSENSE: Flexible FE/BE Sensor Pilot Line for the Internet of Everything" funded by the Electronic Component Systems for European Leadership (ECSEL) Joint Undertaking under Grant Agreement no. 692480. The work is also partially supported by Project "AM4G: Advanced Manufacturing 4th Generation."

\section{References}

[1] R. Bucki and P. Suchánek, "Modelling decision-making processes in the management support of the manufacturing element in the logistic supply chain," Complexity, vol. 2017, Article ID 5286135, 2017.

[2] V. Modrak and Z. Soltysova, "Novel complexity indicator of manufacturing process chains and its relations to indirect complexity indicators," Complexity, vol. 2017, Article ID 9102824, 2017.

[3] J. L. García-Alcaraz, A. A. Maldonado-Macías, S. I. HernándezHernández, J. L. Hernández-Arellano, J. Blanco-Fernández, 
and J. C. Sáenz Díez-Muro, "New product development and innovation in the maquiladora industry: A causal model," Sustainability, vol. 8, no. 8, article no. 707, 2016.

[4] R. E. Haber, R. Haber, A. Alique, and S. Ros, "Application of knowledge-based systems for supervision and control of machining processes," Handbook of software engineering and knowledge engineering, vol. 2, pp. 673-710, 2002.

[5] G. Beruvides, R. Quiza, R. Del Toro, and R. E. Haber, "Sensoring systems and signal analysis to monitor tool wear in microdrilling operations on a sintered tungsten-copper composite material," Sensors and Actuators A: Physical, vol. 199, pp. 165175, 2013.

[6] R. E. Haber and J. R. Alique, "Nonlinear internal model control using neural networks: An application for machining processes," Neural Computing and Applications, vol. 13, no. 1, pp. 47-55, 2004.

[7] R.-E. Precup, P. Angelov, B. S. J. Costa, and M. SayedMouchaweh, "An overview on fault diagnosis and natureinspired optimal control of industrial process applications," Computers in Industry, vol. 74, pp. 1-16, 2015.

[8] R. Calvo, R. D’Amato, E. Gómez, and R. Domingo, "Integration of error compensation of coordinate measuring machines into feature measurement: Part II-experimental implementation," Sensors, vol. 16, no. 10, article no. 1705, 2016.

[9] R. Calvo, R. D’Amato, E. Gómez, and R. Domingo, "Integration of error compensation of coordinate measuring machines into feature measurement: Part I-model development," Sensors, vol. 16, no. 10, article no. 1610, 2016.

[10] G. Beruvides, F. Castaño, R. Quiza, and R. E. Haber, "Surface roughness modeling and optimization of tungsten-copper alloys in micro-milling processes," Measurement: Journal of the International Measurement Confederation, vol. 86, pp. 246-252, 2016.

[11] P. B. Huang, "An intelligent neural-fuzzy model for an inprocess surface roughness monitoring system in end milling operations," Journal of Intelligent Manufacturing, vol. 27, no. 3, pp. 689-700, 2016.

[12] M. Tomov, M. Kuzinovski, and P. Cichosz, "Modeling and prediction of surface roughness profile in longitudinal turning," Journal of Manufacturing Processes, vol. 24, pp. 231-255, 2016.

[13] H. El-Mounayri, Z. Dugla, and H. Deng, "Prediction of surface roughness in end milling using swarm intelligence," in Proceedings of the 2003 IEEE Swarm Intelligence Symposium, SIS 2003, pp. 220-227, usa, April 2003.

[14] N. Liu, S. B. Wang, Y. F. Zhang, and W. F. Lu, "A novel approach to predicting surface roughness based on specific cutting energy consumption when slot milling Al-7075," International Journal of Mechanical Sciences, vol. 118, pp. 13-20, 2016.

[15] M. P. Sealy, Z. Y. Liu, Y. B. Guo, and Z. Q. Liu, "Energy based process signature for surface integrity in hard milling," Journal of Materials Processing Technology, vol. 238, pp. 284-289, 2016.

[16] A. Alique, R. E. Haber, R. H. Haber, S. Ros, and C. Gonzalez, "Neural network-based model for the prediction of cutting force in milling process. A progress study on a real case," in Proceedings of the 2000 IEEE International Symposium on Intelligent Control, pp. 121-125.

[17] R. E. Haber, J. R. Alique, A. Alique, J. Hernández, and R. UribeEtxebarria, "Embedded fuzzy-control system for machining processes: Results of a case study," Computers in Industry, vol. 50, no. 3, pp. 353-366, 2003.

[18] D. M. D’Addona, A. S. Ullah, and D. Matarazzo, "Toolwear prediction and pattern-recognition using artificial neural network and DNA-based computing," Journal of Intelligent Manufacturing, vol. 28, no. 6, pp. 1285-1301, 2015.

[19] J. M. Andújar and A. J. Barragán, "Hybridization of fuzzy systems for modeling and control," RIAI - Revista Iberoamericana de Automatica e Informatica Industrial, vol. 11, no. 2, pp. 127-141, 2014.

[20] W. Pedrycz and K.-C. Kwak, "The development of incremental models," IEEE Transactions on Fuzzy Systems, vol. 15, no. 3, pp. 507-518, 2007.

[21] S.-B. Roh, T.-C. Ahn, and W. Pedrycz, "The refinement of models with the aid of the fuzzy k-nearest neighbors approach," IEEE Transactions on Instrumentation and Measurement, vol. 59, no. 3, pp. 604-615, 2010.

[22] G. Kiswanto, D. L. Zariatin, and T. J. Ko, "The effect of spindle speed, feed-rate and machining time to the surface roughness and burr formation of Aluminum Alloy 1100 in micro-milling operation," Journal of Manufacturing Processes, vol. 16, no. 4, pp. 435-450, 2014.

[23] C.-B. Cheng and E. S. Lee, "Nonparametric fuzzy regression k-NN and kernel smoothing techniques," Computers \& Mathematics with Applications, vol. 38, no. 3, pp. 239-251, 1999.

[24] H.-L. Chen, B. Yang, G. Wang et al., "A novel bankruptcy prediction model based on an adaptive fuzzy k-nearest neighbor method," Knowledge-Based Systems, vol. 24, no. 8, pp. 1348-1359, 2011.

[25] Z. Ming, Z. Feng, and J. Zhengbiao, "A study on speech emotion recognition based on fuzzy K nearest neighbor," International Journal of Multimedia and Ubiquitous Engineering, vol. 10, no. 10, pp. 57-66, 2015.

[26] A. Fiannaca, G. Di Fatta, R. Rizzo, A. Urso, and S. Gaglio, "Simulated annealing technique for fast learning of SOM networks," Neural Computing and Applications, vol. 22, no. 5, pp. 889-899, 2013.

[27] R. A. Rutenbar, "Simulated annealing algorithms: an overview," IEEE Circuits and Devices Magazine, vol. 5, no. 1, pp. 19-26, 1989 (English).

[28] F. Zhao, Y. Hong, D. Yu, Y. Yang, Q. Zhang, and H. Yi, “A hybrid algorithm based on particle swarm optimization and simulated annealing to holon task allocation for holonic manufacturing system," The International Journal of Advanced Manufacturing Technology, vol. 32, no. 9-10, pp. 1021-1032, 2007.

[29] F. Penedo, R. E. Haber, A. Gajate, and R. M. Del Toro, "Hybrid incremental modeling based on least squares and fuzzy K$\mathrm{NN}$ for monitoring tool wear in turning processes," IEEE Transactions on Industrial Informatics, vol. 8, no. 4, pp. 811-818, 2012.

[30] J. Derrac, F. Chiclana, S. García, and F. Herrera, "Evolutionary fuzzy k-nearest neighbors algorithm using interval-valued fuzzy sets," Information Sciences, vol. 329, pp. 144-163, 2016.

[31] M. Almaraashi, R. John, A. Hopgood, and S. Ahmadi, "Learning of interval and general type-2 fuzzy logic systems using simulated annealing: Theory and practice," Information Sciences, vol. 360, pp. 21-42, 2016.

[32] D.-Y. Sun and P.-M. Lin, "The solution of time optimal control problems by simulated annealing," Journal of Chemical Engineering of Japan, vol. 39, no. 7, pp. 753-766, 2006.

[33] F. Castaño, R. E. Haber, R. M. Del Toro, and G. Beruvides, "Application of hybrid incremental modeling for predicting surface roughness in micromachining processes," in Proceedings of the 2014 IEEE Symposium on Computational Intelligence for Engineering Solutions, CIES 2014, pp. 54-59, USA, December 2014. 
[34] Geometrical Product Specifications (GPS) - Indication of surface texture in technical product documentation, 2002.

[35] M.-Y. Wang and H.-Y. Chang, "Experimental study of surface roughness in slot end milling AL2014-T6," The International Journal of Machine Tools and Manufacture, vol. 44, no. 1, pp. 5157, 2004.

[36] M. Correa, C. Bielza, and J. Pamies-Teixeira, "Comparison of bayesian networks and artificial neural networks for quality detection in a machining process," Expert Systems with Applications, vol. 36, no. 3, pp. 7270-7279, 2009. 


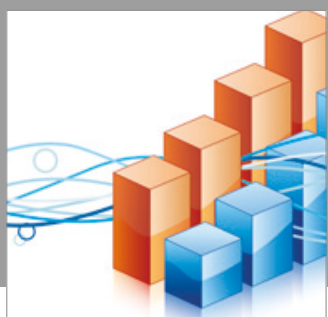

Advances in

Operations Research

vatersals

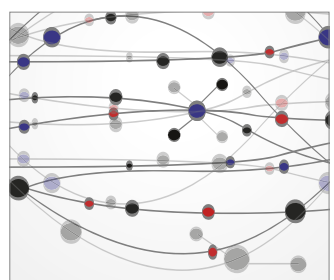

\section{The Scientific} World Journal
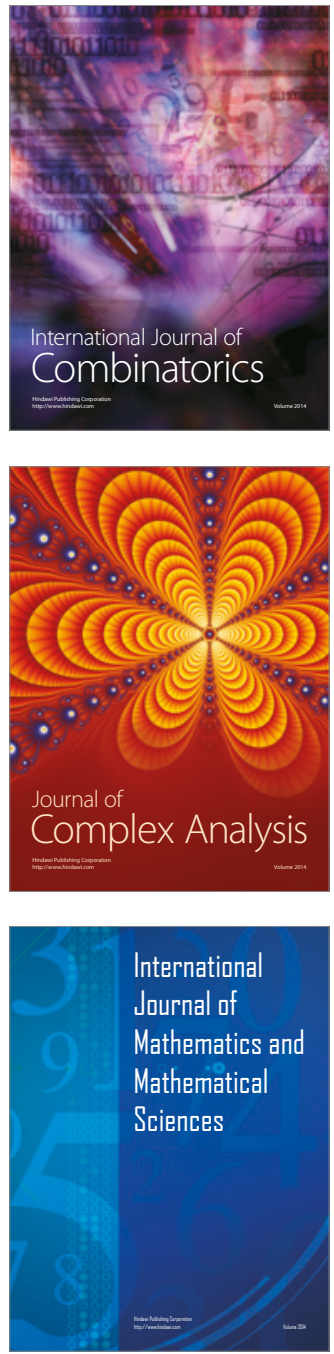
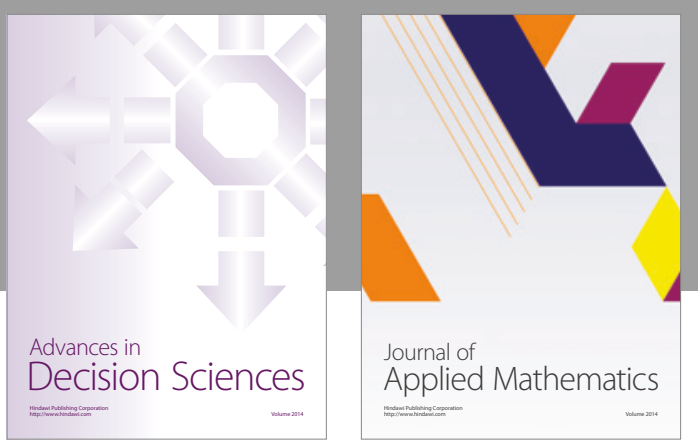

Algebra

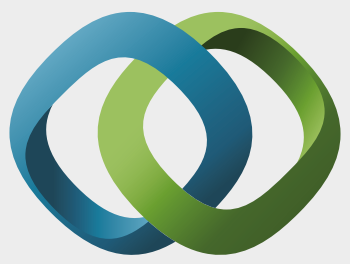

\section{Hindawi}

Submit your manuscripts at

https://www.hindawi.com
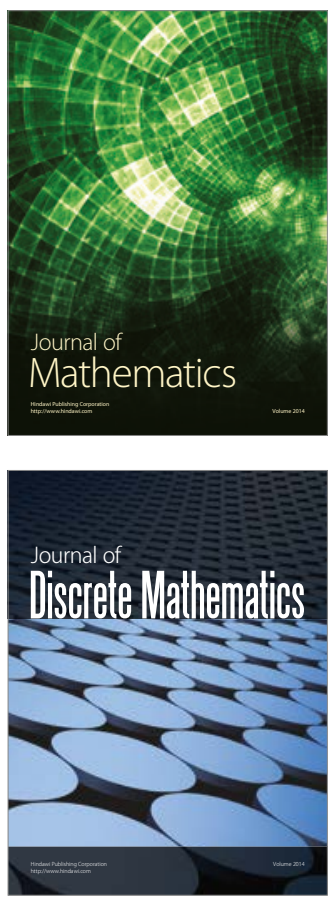

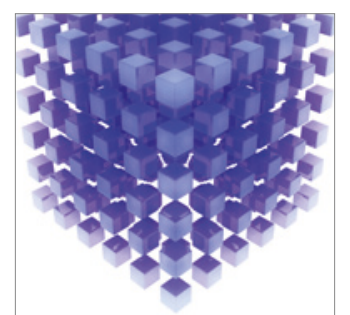

Mathematical Problems in Engineering
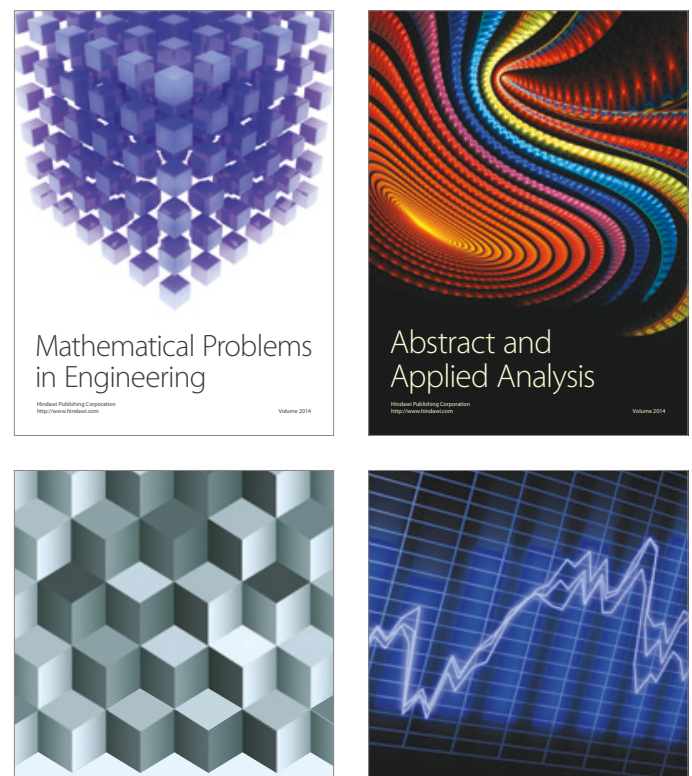

Journal of

Function Spaces

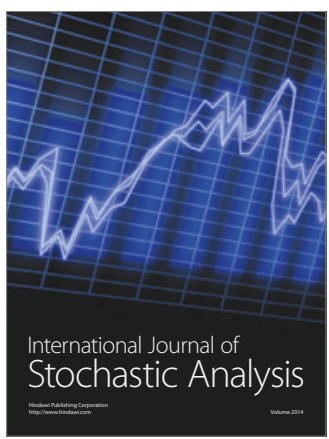

Probability and Statistics
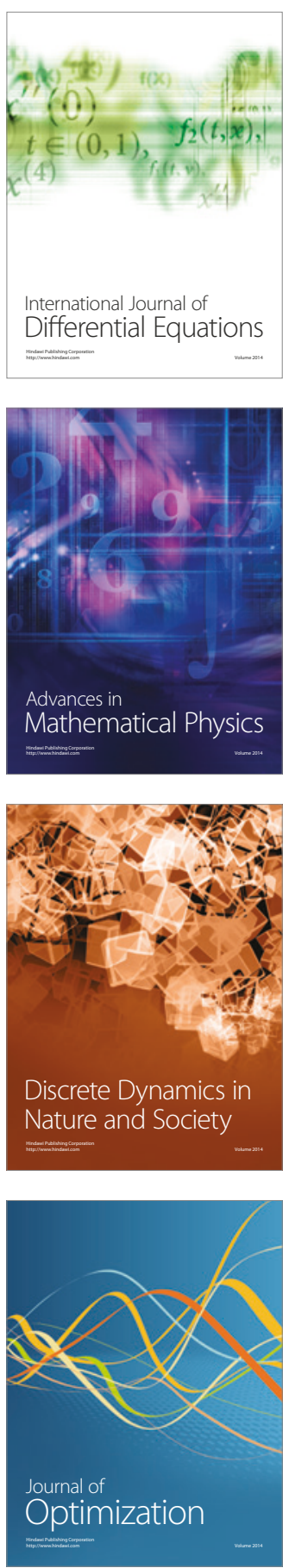